\title{
Klimaanpassung von Logistikstandorten - eine Szenarienanalyse
}

\author{
Felix Bücken' • Helga Kanning²
}

Eingegangen: 22. Februar 2021 / Überarbeitet: 23. April 2021 / Angenommen: 15. Mai 2021 / Online publiziert: 23. Juni 2021

(c) Der/die Autor(en) 2021

\section{Zusammenfassung}

Für die Entwicklung von Klimaanpassungsstrategien sind Logistikstandorte aus zwei Perspektiven bedeutsam: Einerseits sind Logistikunternehmen selbst aufgrund ihrer großen Liegenschaften und des hohen Versiegelungsgrads besonders von den Folgen des Klimawandels betroffen, andererseits erhöhen sie durch ihre umfassenden Flächenversiegelungen die Vulnerabilität der Kommunen, in denen sie angesiedelt sind. Sowohl Logistikunternehmen als auch Kommunen sind daher gefragt, klimaangepasste Gewerbeflächen zu entwickeln. Mithilfe einer kritisch reflektierten Szenarienanalyse zeigt dieser Beitrag verschiedene Positionen beteiligter Akteure (Logistikunternehmen, Kommunen, Intermediäre) auf und identifiziert Hemmnisse sowie Konfliktfelder zur Anpassung an Klimafolgen. Dazu werden 3 Szenarien vorgestellt, die verschiedene Gestaltungs- und Entwicklungsperspektiven der Anpassung und Flächennutzung illustrieren. Aus der Diskussion der Szenarien im Rahmen von 3 Workshops lassen sich 4 zentrale Handlungsfelder ableiten, die für die Anpassung von Logistikstandorten besonders bedeutsam sind: Interkommunale Zusammenarbeit, intrakommunale Herausforderungen, wie z.B. mangelnde personelle Ressourcen in den Verwaltungen, mehr Zukunftsorientierung der Logistik sowie Sensibilisierung und Kommunikation. Dabei wird die Verantwortung für die Umsetzung geeigneter Maßnahmen in Kooperation durch Unternehmen, Kommunen und Intermediäre gesehen.

Schlüsselwörter Klimarisiken · Transportwirtschaft · Gewerbeflächen · Landmanagement · Workshops

\begin{abstract}
Logistics locations are significant for the development of climate change adaptation strategies from two perspectives: on the one hand, logistics companies themselves are particularly affected by the consequences of climate change owing to their large properties and the high degree of sealing; on the other hand, they increase the vulnerability of the municipalities in which they are located, with their extensive land sealing. Both logistics companies and municipalities are therefore asked to develop climate-adapted commercial areas. By means of a critically reflected scenario analysis, this article shows the different positions of the actors involved (logistics companies, municipalities, intermediaries) and identifies areas of conflict in adapting to the effects of climate change. To this end, three scenarios are presented that illustrate different design and development perspectives for adaptation and land use. From the discussion of the scenarios in three workshops, four central fields of action can be derived that are particularly significant for the adaptation of logistics locations: inter-communal cooperation, intra-communal challenges, such as a lack of human resources in administrations, more future orientation of logistics, as well as sensitization and communication. In this context, responsibility for the implementation of appropriate measures is seen in cooperation by companies, municipalities, and intermediaries.
\end{abstract}

Keywords Climate risks $\cdot$ Transport industry $\cdot$ Commercial areas $\cdot$ Land management $\cdot$ Workshops

Felix Bücken

felix.buecken@uos.de

Helga Kanning

kanning@sustainify.de
1 Institut für Geographie, Universität Osnabrück, Osnabrück, Deutschland

2 sustainify Institut für nachhaltige Forschung, Bildung, Innovation, Hannover, Deutschland 


\section{Klimarisiken für Logistikstandorte und Kommunen}

Obwohl sich das globale Klima bereits zum jetzigen Zeitpunkt messbar verändert hat, werden die Folgen des Klimawandels noch immer mit Ereignissen in ferner Zukunft verbunden (Marx 2017). Gleichwohl äußert sich der Klimawandel in Deutschland durch häufiger und drastischer werdende Extremwetterereignisse (GDV 2019). Dabei tragen die Folgen des Klimawandels zu einer Zunahme von Risiken bei - nicht nur auf individueller Ebene, sondern auch und vor allem in Bezug auf die Zukunftsfähigkeit von Geschäftsmodellen, Infrastrukturen und Ökonomien (Averchenkova et al. 2016).

Auch Unternehmen befassen sich zunehmend mit der Frage, welche Konsequenzen Klimarisiken auf ihren Unternehmenserfolg haben. Neben Störungen in Lieferketten stehen vor allem extremwetterbedingte Schäden an Unternehmensstandorten und die daraus entstehenden Geschäftsrisiken in ihrem Fokus (Co2ncept Plus 2020). Physische Schäden verursachen - neben unmittelbaren Kosten für Reparaturen - Beeinträchtigungen von Prozessen und somit, z. B. im Fall von Lieferverzögerungen, indirekte Kosten (World Bank 2020). McKinnon und Kreie (2010) weisen darauf hin, dass die tatsächliche Betroffenheit von Unternehmensstandorten von den Eigenschaften der genutzten Liegenschaften und ihrer Lokalisierung abhängt. Auch besteht ein unmittelbarer Zusammenhang zwischen der Betroffenheit eines Unternehmens gegenüber Klimarisiken und der Branche, in der es agiert: Haben Unternehmen vergleichbare Geschäftsziele und unterliegen ähnlichen marktbezogenen und regulativen Zwängen, führt dies zu vergleichbaren Strukturen (Weinhofer und Busch 2013).

Logistikbetriebe verfügen zum Zwecke der Lagerung und des Umschlags von Gütern über überdurchschnittlich große Liegenschaften (Garbe und Hempel 2015). Daraus ergibt sich eine erhöhte Anfälligkeit der Betriebsstandorte gegenüber Extremwetter. Die versiegelten Flächen, die für Verkehre auf dem Gelände sowie für Lagerungen und den ruhenden Verkehr genutzt werden (vgl. Abb. 1), sind oftmals sensibel für Hitzeschäden. Auch heizen sich Büroräume, Läger und Fahrzeuge unter hohen Temperaturen stark auf, was bei unzureichender Klimatisierung zu einem Leistungsabfall des Personals führen kann. Starkregenereignisse steigern ferner das Risiko plötzlicher und intensiver Überflutungen der Grundstücke (Weller et al. 2016). Um den Klimarisiken etwas entgegenzusetzen, sind Unternehmen gefragt, Klimaanpassung im Sinne des ,process of adjustment to actual or expected climate and its effects" (IPCC 2014, S. 76) zu betreiben und zielgenaue regionale Lösungen für konkrete Beeinträchtigungen herzustellen (Marx 2017).

Für Kommunen spielt die Entwicklung klimaangepasster Logistikstandorte ebenfalls eine wichtige Rolle. Flächen-

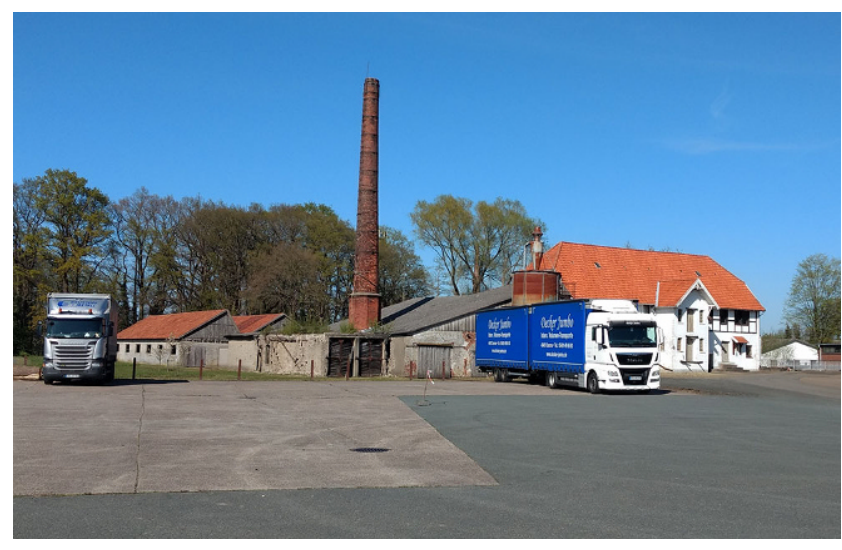

Abb. 1 Versiegelte Freifläche. Quelle: Eigene Aufnahme

versiegelungen, die besonders bei Logistikstandorten in großflächigem Ausmaß typisch sind, minimieren natürliche Ökosystemleistungen, wie regulierende Boden- sowie Arten- und Biotopfunktionen, und erhöhen damit die Vulnerabilität gegenüber Klimawandelphänomenen, wie Starkregen, Hitze und Sturm. In Ballungs- und infrastrukturell bereits gut erschlossenen Siedlungsräumen, in denen sich Logistiknutzungen bevorzugt ansiedeln, beeinflusst das Aufheizen großer versiegelter Flächen und konventioneller Lagerhallen zudem die klimatischen Bedingungen in benachbarten Siedlungsbereichen. Den für den Luftaustausch erforderlichen Kaltluftschneisen kommt damit zusätzliche Bedeutung zu. Die ohnehin stark konkurrierenden Nutzungsansprüche an Flächen (Wohnen, Gewerbe, Verkehr, etc.) werden hierdurch weiter verschärft. Aus Sicht der Planung ist es daher effektiv, Logistiknutzungen besonders in den Fokus zu nehmen und darauf hinzuwirken, großflächige Flächenversiegelungen möglichst zu minimieren bzw. die Standorte so klimaangepasst wie möglich zu gestalten. Mittels verbindlicher Festsetzungen in Bebauungsplänen können Kommunen eine Berücksichtigung von Maßnahmen zur Klimaanpassung bei der Entwicklung von Gewerbegebieten bewirken und sicherstellen, dass eine Anpassung vorsorglich bereits im Rahmen der Projektentwicklung berücksichtigt wird. In der Konsequenz erfordert die Klimaanpassung, neben der Initiative der Wirtschaft, ein verantwortungsvolles Flächenmanagement seitens kommunaler Akteure. Offen ist, welche Rolle Intermediäre, wie Wirtschaftsförderungen, die zwischen Unternehmen und kommunaler Verwaltung vermitteln, in diesem Handlungsfeld spielen können. Ihnen wird seit Kurzem zunehmende Bedeutung, z.B. hinsichtlich eines nachhaltigen Gewerbeflächenmanagements, zugeschrieben (Hallmann 2020).

Die unterschiedlichen Blickwinkel auf das Thema Klimaanpassung stellen hohe Ansprüche an die Kooperationsfähigkeiten der Akteure und an die Transparenz ihrer jeweiligen Anforderungen an eine nachhaltige Entwicklung von Gewerbeflächen. Ziel dieses Beitrags ist es daher, ver- 
schiedene Positionen beteiligter Akteursgruppen (Logistikunternehmen, Kommunen und intermediäre Akteure) sowie Hemmnisse zur Anpassung an Klimafolgen, Konfliktfelder und mögliche Synergien aufzuzeigen. Die hierzu verwendete Szenarienanalyse wird zudem abschließend einer kritischen Betrachtung hinsichtlich ihrer Eignung zur Identifikation verschiedener Interessen und relevanter Konfliktfelder unterzogen.

\section{Szenarioanalyse}

Im Rahmen des vom Bundesumweltministerium geförderten Projekts „KlimaLogis - Klimaangepasste Logistik Ein Pilotprojekt im Landkreis Osnabrück“" wurden im Zeitraum September bis Oktober 2020 drei Workshops durchgeführt, die sich jeweils an Mitarbeiter*innen aus dem Bereich der Planung, der Wirtschaftsförderung und Vertreter*innen regional ansässiger Logistikunternehmen richteten. In der Region Osnabrück ist die Logistik einer der bedeutenden, wachsenden Wirtschaftszweige. Die Bundesvereinigung Logistik sieht die Region als „Top Logistikstandort mit engagierten und nachhaltig agierenden Unternehmen, guter Infrastruktur, hervorragenden Mitarbeitern und Hochschulen“ (Vallée 2014, S. 3). Die günstige Kombination aus harten und weichen Standortfaktoren erzeugt eine hohe Nachfrage nach Grundstücken für logistische Nutzungen, woraus ein zunehmender Flächenmangel resultiert. Neben der allgemein geringen Flächenverfügbarkeit, verringern die Eigenschaften von Logistiknutzungen (hoher Flächenverbrauch und Flächenversiegelung, geringe Arbeitsplatzdichte, Emissionen durch Verkehre, unattraktive Architektur) ihre Akzeptanz. In der Folge entsteht in der Region Osnabrück eine Diskrepanz zwischen der wachsenden Bedeutung eines Wirtschaftszweigs und einer zunehmend ablehnenden Haltung seitens der Öffentlichkeit (Garbe und Hempel 2015).
Im Zentrum der im KlimaLogis-Projekt durchgeführten Workshopveranstaltungen standen drei durch das Sustainify-Institut entwickelte Szenarien, die verschiedene Gestaltungs- und Entwicklungsperspektiven abbilden (Kanning und Richter-Harm 2020). Die im Folgenden in den Szenarien dargestellten Entwicklungspfade und Zukunftsbilder für einen mittelfristigen Zeithorizont bis zum Jahr 2030 basieren auf Erfahrungswissen, Literaturrecherchen sowie dem im vorherigen Projektverlauf durchgeführten Dialog mit Unternehmen und öffentlichen Akteuren.

Szenario 1 (Trend, Abb. 2): Die Logistikunternehmen reagieren nach dem Eintreten konkreter Klimafolgen in Einzelfällen mit Anpassungsmaßnahmen auf ihren Betriebsgeländen (z.B. Verbesserung der Entwässerung, Verschattung von Büroflächen). Die Unternehmen vertrauen darauf, dass die Festsetzungen in Bebauungsplänen etwaige Klimarisiken ausreichend berücksichtigen. Mit der Anpassung von Bestandsimmobilien befassen sich nur wenige Unternehmen, regelmäßig eintretende Beschädigungen werden pragmatisch behoben. Vom Landkreis werden u.a. Beratungen für Unternehmen angeboten und damit Anpassungsmaßnahmen auf Gewerbeflächen und an Gebäuden erzielt. Ein nachhaltiges Flächenmanagement sorgt punktuell für Ansiedlungen auf Bestandsflächen, eine maßgebliche Reduktion der Flächenversiegelung wird aber nicht erreicht. Die Kommunen im Landkreis agieren unterschiedlich: Einige legen Anpassungsmaßnahmen in Bebauungsplänen fest, andere verzichten darauf.

Szenario 2 (Worst Case, Abb. 3): Die Unternehmen treffen nur unabdingbare Anpassungsmaßnahmen unmittelbar nach dem Eintreten von Schäden. Die Verantwortung für Anpassungsmaßnahmen wird ausschließlich bei den Kommunen gesehen. Vom Landkreis gehen keine Impulse für die Klimaanpassung aus. Die Kommunen im Landkreis legen aufgrund ihrer Konkurrenzsituation keine Klimaanpassungsmaßnahmen in Bebauungsplänen fest. Um Neuansiedlungen zu erleichtern und Gewerbesteuereinnahmen zu
Abb. 2 Trend-Szenario (Kanning und Richter-Harm 2020, S. 14-15)

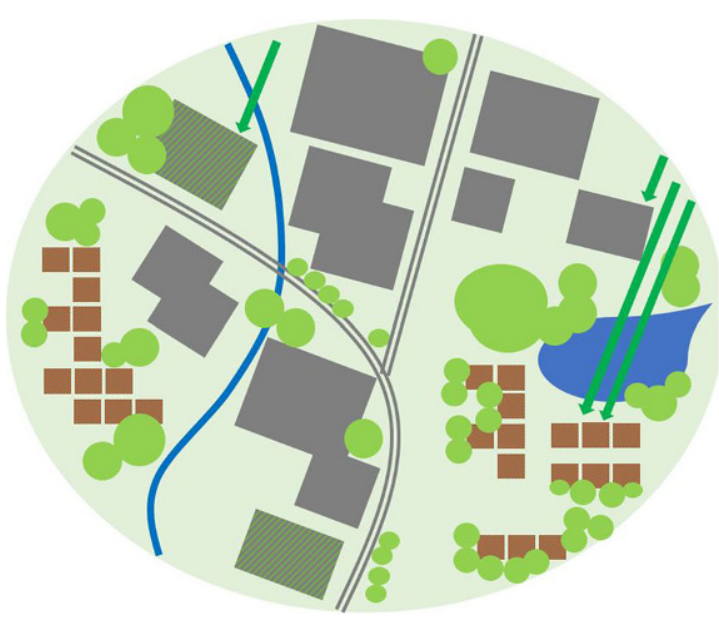


Abb. 3 Worst-Case-Szenario (Kanning und Richter-Harm 2020, S. 16-17)

Abb. 4 Best-Case-Szenario (Kanning und Richter-Harm 2020, S. 17-19)
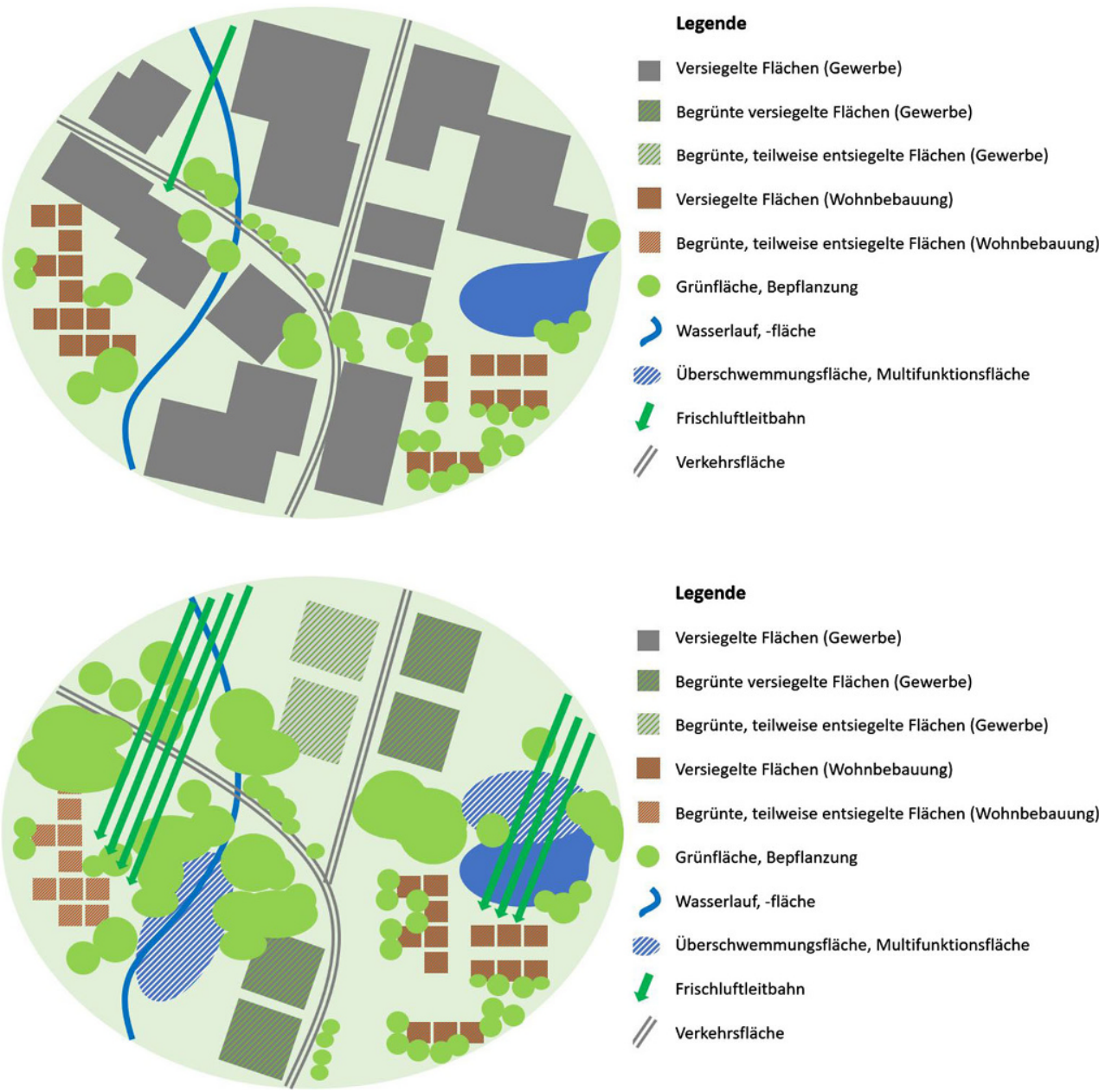

maximieren, werden Flächen ohne Restriktionen entwickelt und vermarktet. Die Flächenausweisungspolitik der Kommunen erfolgt unkoordiniert und ohne überörtliche strategische Ausrichtung.

Szenario 3 (Best Case, Abb. 4): Die Unternehmen haben präventiv Anpassungsmaßnahmen an ihren Standorten entwickelt und umgesetzt. Intermediäre, wie die Wirtschaftsförderung, Kammern oder Branchenverbände, handeln proaktiv und engagieren sich für Wissenstransfer und Qualifizierung im Bereich der Klimaanpassung. Ein regionales Logistikflächenkonzept dient als abgestimmte verlässliche Grundlage zur Planung und Entwicklung neuer Logistikflächen. Über Anreizprogramme werden Klimaanpassungsmaßnahmen auf Bestandsflächen gefördert. Zudem hat sich der Flächenbedarf der Unternehmen durch neue Nutzungskonzepte und den Einsatz digitaler Technologien verringert.

\section{Ergebnisse}

Das Trend-Szenario wird von den Planer*innen als realitätsnah wahrgenommen. Nur die Haltung der Logistikunternehmen wird angezweifelt: Dass Betriebe ihre Immobilien eigeninitiativ anpassen, wird aus ihrer Sicht nur in wenigen Fällen vorkommen. Das Worst-Case-Szenario wird von der Akteursgruppe Planung als zukünftiger Zustand ausgeschlossen, da Fragen der Klimaanpassung heute bereits in der Flächennutzungs- und Bebauungsplanung berücksichtigt werden. Für die Wirtschaftsförderung zeigt Szenario 2 eine Form der Gewerbegebietsentwicklung, die nach heutigem Kenntnisstand unattraktive Unternehmensstandorte erzeugt, die meist schwer zu vermarkten sind. Von den Unternehmensvertreter*innen wird das Szenario aufgrund des heute bereits vorhandenen Umfangs an Regulierungen als nicht realistisch erachtet. Das Best-Case-Szenario ist nach Einschätzung der Planer*innen ein positives Entwicklungsziel, das derzeit verfolgt wird. Die Wirtschaftsförder*innen erachten dieses Szenario ebenfalls als wünschenswert, äuBern zugleich jedoch starke Zweifel an der Realisierbarkeit. 
Begründet wird dies nicht mit mangelnder Investitionsbereitschaft der Unternehmen, sondern mit der Einstellung der Politik. Die Unternehmen hinterfragen die Wirtschaftlichkeit dieses Szenarios. Sie befürchten ein negatives KostenNutzen-Verhältnis und Wettbewerbsverzerrungen.

Als entscheidendes Problemfeld bei der Gestaltung eines klimaangepassten Flächenmanagements wurden interkommunale Konflikte und eine unzureichende Kooperationsbereitschaft zwischen Gebietskörperschaften identifiziert. Die Planer*innen betonen hierbei die Notwendigkeit einer gemeinsamen Flächen- und Ansiedlungspolitik für logistische Nutzungen sowie einer überörtlichen Flächenbevorratung.

Neben Konflikten zwischen Kommunen sind auch Zielkonflikte innerhalb der jeweiligen Verwaltungen hinderlich für die Anpassung an die Folgen des Klimawandels. Die Planer*innen weisen darauf hin, dass verschiedene Fachressorts innerhalb der Verwaltungen unterschiedliche Zielsetzungen verfolgen, was eine gemeinsame kommunale Praxis erschwert. Des Weiteren sind die Ziele der Verwaltung häufig nicht mit den Vorstellungen der Politik kompatibel. Ähnlich wie Unternehmen verfolgen politische Entscheidungsträger*innen häufig das Prinzip der Gewinnmaximierung. Umfassende Regulierungen zur Klimaanpassung stehen dieser Maxime entgegen, da die Vorstellung vorherrscht, Unternehmen würden durch Restriktionen abgeschreckt und die zur Vermarktung verfügbare Fläche reduziert. Damit geht die Befürchtung einher, aus der vorhandenen Fläche nicht das Optimum an Arbeitsplätzen und Gewerbesteuer generieren zu können. Darüber hinaus wirken mangelnde personelle Ressourcen in den Kommunen hemmend auf die Implementierung von Klimaanpassungsmaßnahmen.

Auch in der Wirtschaft bestehen Faktoren, die den Weg in Richtung Szenario 3 erschweren. So bescheinigen alle Akteursgruppen der Logistikbranche eine unterdurchschnittlich ausgeprägte Zukunftsorientierung. Vonseiten der Planer*innen bezieht sich diese Kritik vornehmlich auf die sporadische Umsetzung von Konzepten zur Reduktion des Flächenverbrauchs, die als dringend erforderlich angesehen werden. Die Vertreter*innen der Unternehmen erklären die gegenwartszentrierten Logiken ihrer Branche vor allem mit der Notwendigkeit der flexiblen Anpassung an sich stetig verändernde Kundenwünsche. Die Unternehmen reagieren lediglich auf Anforderungen und nehmen selbst keine gestaltende Position ein. Dabei erschwert die geringe Umsatzrendite in der Logistik die Implementierung von Klimaanpassungsmaßnahmen. Die Ertüchtigung von Bestandsimmobilien und die Investition in klimaangepasste Neubauprojekte setzten finanzielle Ressourcen voraus, über die Logistikunternehmen in geringerem Umfang verfügen als Unternehmen des produzierenden Gewerbes. Ferner wurde deutlich, dass Klimaanpassung als wichtige zukünftige Zielvorstellung in einem größeren Kontext gesehen werden muss. Problematisch ist vor allem, dass dieses Ziel bisher kaum präsent ist und vielen Verantwortlichen grundlegendes Wissen und Erfahrungen in diesem Themenfeld fehlen. Klimaanpassung ist nach Auffassung der Wirtschaftsförder*innen als solitäres Thema nicht weit genug entwickelt, als dass es möglich wäre, es gegenüber der Zielgruppe Unternehmen attraktiv zu vermitteln. Vielmehr ist es notwendig, es im Zusammenhang mit anderen Themen im Umweltkontext zu platzieren und so einen Fortschritt herbeizuführen.

Zusammenfassend lassen sich aus den Szenarienworkshops 4 zentrale Handlungsfelder ableiten:

1. Interkommunale Zusammenarbeit: Hemmnisse für die Umsetzung einer klimaangepassten Flächenpolitik beruhen maßgeblich auf der unzureichenden Kooperationsbereitschaft zwischen den Kommunen. Es besteht Einigkeit dahingehend, dass eine gemeinsame Flächen- und Ansiedlungspolitik für logistische Nutzungen notwendig ist.

2. Intrakommunale Herausforderungen: Innerhalb der kommunalen Verwaltungen und zwischen Verwaltung und Politik bestehen unterschiedliche Auffassungen darüber, welche Relevanz Klimaanpassung als Entwicklungsziel beizumessen ist. Für den Ausgleich bestehender Zielkonflikte sind zusätzliche personelle Ressourcen erforderlich.

3. Zukunftsorientierung der Logistik: Logistikunternehmen befassen sich bislang kaum mit Konzepten zur Reduktion des Flächenverbrauchs oder zur klimaangepassten Umgestaltung von Immobilien. Die konstatierte mangelnde Zukunftsorientierung erfordert effektive Anreize, deren Management zum Teil in den Verantwortungsbereich der Kommunen fällt. So können z. B. Wirtschaftsförderungen Beratungen zu Förderoptionen für Investitionen in bauliche Maßnahmen durchführen.

4. Sensibilisierung und Kommunikation: Eine grundlegende Sensibilisierung für das Thema und die Vermittlung von Erfahrungen und Wissen zu konkreten Anpassungsmaßnahmen ist erforderlich, um die Flächenentwicklung und -nutzung grundlegend zu verändern. Um mehr Interesse bei den Unternehmen hervorzurufen, ist eine Verknüpfung an etablierte Themen von unternehmerischer Relevanz (z. B. Risikomanagement) empfehlenswert.

Für die Umsetzung geeigneter Maßnahmen erscheint eine kooperative Form der Verantwortungsübernahme zielführend. Dieses wird seitens der Wirtschaftsförderung und von Teilen der Wirtschaft befürwortet. Die Wirtschaftsförder*innen betonen die Bedeutung der Eigeninitiative der Betriebe, die anhand einheitlicher Regulierungen kanalisiert werden muss. Die Aussagen der Unternehmensvertreter*innen bestätigen, dass die Entwicklung nachhaltiger Strategien, die betriebliche Ziele mit Klimaanpassung in 
Einklang bringen, in den Verantwortungsbereich der Wirtschaft fällt. Aufgabe der Kommunen sei es, notwendige Klimaanpassungsmaßnahmen mittels planungsrechtlicher Instrumente einzufordern. Auf intermediäre Organisationen, die eine Brückenfunktion zwischen Wirtschaft und öffentlichen Belangen erfüllen, entfällt nach Meinung der Wirtschaftsförder*innen die Aufgabe der Sensibilisierung für Klimaanpassung als relevantes zukünftiges Handlungsfeld. Hier gilt es, bisher nicht hinreichend vorhandene Informationen zur Klimaanpassung für die Unternehmen bereitzustellen und zur Aufklärung in diesem Themenfeld beizutragen.

\section{Methodenreflexion}

Die vorliegenden Ergebnisse verdeutlichen, dass die Methode des Szenarienworkshops zur Identifikation divergierender Interessen und relevanter Konfliktfelder überaus zielführend ist. Dabei ist die inhaltliche Offenheit der Diskussion die besondere Stärke der Herangehensweise: Obwohl durch die Szenarien ein Diskussionsrahmen vorgegeben wird, zeichnen sich die Äußerungen der Akteure durch ein breites thematisches Spektrum aus. Auf diese Weise konnten Handlungsfelder identifiziert werden, die ohne ein Szenario als Anreiz und gedanklichen Ankerpunkt nicht aufgekommen wären. Gleichwohl leistet die Methode in ihrer hier durchgeführten Form keinen direkten Beitrag für den Ausgleich von Interessen. Da die Akteursgruppen getrennt voneinander mit den Szenarien konfrontiert wurden, konnte keine interessenübergreifende Diskussion zu möglichen Lösungen angeregt werden. In einem weiteren Schritt sollten daher die identifizierten Handlungsfelder akteursübergreifend erörtert werden.

\section{Fazit}

- Für Logistikunternehmen ist es notwendig, Flächen und Gebäude anzupassen, Sachschäden zu vermeiden und Nutzer*innen der Immobilien zu schützen - letztlich mit dem Ziel, ihren wirtschaftlichen Erfolg dauerhaft zu sichern. Allerdings lässt sich die Anpassung an sich verändernde Rahmenbedingungen nicht ausschließlich durch das Engagement der Wirtschaft erreichen. Politik und Verwaltung - insbesondere auf kommunaler Ebene tragen die Verantwortung für die Konzeption klimaangepasster Flächennutzungen, mit der Risiken reduziert und dauerhafte Standortsicherheit hergestellt werden können. Die durchgeführten Szenarienworkshops zeigen, dass seitens der Unternehmen grundsätzlich eine passive Haltung gegenüber Regulierungen vorherrscht. Auflagen werden nicht begrüßt, wohl aber akzeptiert, sofern sie nicht zu Wettbewerbsverzerrungen beitragen und pragmatisch umgesetzt werden können. Hieraus ergeben sich hohe Anforderungen an die Entwicklung und Planung von Gewerbeflächen, die eine Verbesserung der interkommunalen Kooperation und intrakommunalen Abstimmung voraussetzen.

- Dass die Anpassung an Klimafolgen ein neues Handlungsfeld ist, wurde durch den von allen Akteursgruppen zum Ausdruck gebrachten erhöhten Kommunikationsbedarf deutlich. Aktuell mangelt es allen Akteuren an Wissen zu konkreten Risiken, zum langfristigen Nutzen von Anpassungsmaßnahmen und zur potenziellen Akzeptanz von Festsetzungen in Plänen und zu den daraus resultierenden Konsequenzen für die Vermarktbarkeit von Flächen. Intermediäre Akteure können hier eine zentrale Rolle einnehmen und sowohl Transparenz zwischen den Anforderungen von Unternehmen und den Gestaltungsspielräumen von Kommunen herstellen als auch grundlegendes Wissen zu den Wirkungen von Anpassungsmaßnahmen bereitstellen. Aufgabe der angewandten Forschung ist es nun, Erkenntnisse über diese Wirkzusammenhänge und ihr Kosten-Nutzen-Verhältnis zu erlangen und den relevanten Akteuren im Sinne der Verbesserung ihrer Entscheidungsgrundlage zur Verfügung zu stellen.

Funding Open Access funding enabled and organized by Projekt DEAL.

Open Access Dieser Artikel wird unter der Creative Commons Namensnennung 4.0 International Lizenz veröffentlicht, welche die Nutzung, Vervielfältigung, Bearbeitung, Verbreitung und Wiedergabe in jeglichem Medium und Format erlaubt, sofern Sie den/die ursprünglichen Autor(en) und die Quelle ordnungsgemäß nennen, einen Link zur Creative Commons Lizenz beifügen und angeben, ob Änderungen vorgenommen wurden.

Die in diesem Artikel enthaltenen Bilder und sonstiges Drittmaterial unterliegen ebenfalls der genannten Creative Commons Lizenz, sofern sich aus der Abbildungslegende nichts anderes ergibt. Sofern das betreffende Material nicht unter der genannten Creative Commons Lizenz steht und die betreffende Handlung nicht nach gesetzlichen Vorschriften erlaubt ist, ist für die oben aufgeführten Weiterverwendungen des Materials die Einwilligung des jeweiligen Rechteinhabers einzuholen.

Weitere Details zur Lizenz entnehmen Sie bitte der Lizenzinformation auf http://creativecommons.org/licenses/by/4.0/deed.de.

\section{Literatur}

Averchenkova A, Crick F, Kocornik-Mina A, Leck Surminski HS, Leck Surminski HS (2016) Multinational and large national corporations and climate adaptation: are we asking the right questions? A review of current knowledge and a new research perspective. Clim Change 7(4):517-536

Co2ncept Plus - Verband der Wirtschaft für Emissionshandel und Klimaschutz e. V. (2020) Klimarisikomanagement 2050 - Betrieb- 
liche Klimarisikostrategie Step-by-Step entwickeln. Co2ncept Plus, München

Garbe C, Hempel J (2015) Die Zukunft der Logistikimmobilie. In: Voß PH (Hrsg) Logistik - eine Industrie, die (sich) bewegt. Springer, Wiesbaden, S 153-166

GDV Gesamtverband der Deutschen Versicherungswirtschaft e.V. (2019) Naturgefahrenbilanz 2019. https://www.gdv.de/de/medien/ aktuell/schaeden-von-3-2-milliarden-euro-durch-stuerme--hagelund-starkregen--54750. Zugegriffen: 8. Febr. 2021

Hallmann I (2020) Ansätze einer nachhaltigen Wirtschaftsförderung auf kommunaler Ebene. In: Stember J, Vogelgesang M, Pongratz $\mathrm{P}$ (Hrsg) Handbuch Innovative Wirtschaftsförderung. Springer, Wiesbaden, S 1-18

IPCC (2014) Climate Change 2014. Synthesis Report. Contribution of Working Groups I, II and III to the Fifth Assessment Report of the Intergovernmental Panel on Climate Change. IPCC, Genf
Kanning H, Richter-Harm B (2020) Klimaangepasste Logistik - Szenarien Landmanagement. sustainify $\mathrm{GmbH}$, Hannover

Marx A (2017) Klimaanpassung in Forschung und Politik. Springer, Wiesbaden

McKinnon A, Kreie A (2010) Adaptive logistics: preparing logistical systems for climate change. https://citeseerx.ist.psu.edu/viewdoc/ download?doi=10.1.1.408.7740\&rep=rep1\&type=pdf. Zugegriffen: 8. Febr. 2021

Vallée F (2014) Die Logistikregion Münster/Osnabrück auf dem Weg nach oben! BVL Regionalgruppe, Münster

Weinhofer G, Busch T (2013) Corporate strategies for managing climate risks. Bus Strat Env 22(2):121-144

Weller B, Fahrion M-S, Horn S, Naumann T, Nikolowski J (2016) Baukonstruktion im Klimawandel. Springer, Wiesbaden

World Bank (2020) Resilient industries. Competitiveness in the face of disasters. World Bank, Washington, DC 